\title{
The Simulation and Performance Evaluation of Adaptive Algorithm of Image Comparison in Correlation-Extreme Navigation Systems
}

\author{
NataliiaYeromina ${ }^{1}$, Sergey Petrov $^{2}$, Yurii Samsonov ${ }^{3}$, Sergii Pisarevskiy $^{4}$, SergiyKaplun $^{5}$, IhorVlasenko $^{6}$ \\ ${ }^{1}$ Department of Electronic Computers, Kharkiv National University of Radio Electronics, Ukraine, Kharkiv, \\ nataliia.yeromina@nure.ua \\ ${ }^{2}$ Department of Physics, Electrical Engineering and Power Engineering, Ukrainian Engineering Pedagogics \\ Academy, Ukraine, psv_topol@ukr.net \\ ${ }^{3}$ National Academy of the National Guard of Ukraine, Kharkiv, Ukraine, yuri1978samsonov@gmail.com \\ ${ }^{4}$ Department of Technical and Logistics, National Academy of the National Guard of Ukraine, Ukraine, \\ psv021180@ukr.net \\ ${ }^{5}$ National Academy of National Guard of Ukraine, Ukraine, serj_kap@ukr.net \\ ${ }^{6}$ Kharkiv National University of Internal Affairs, Ukraine, Vlasenko.18@gmail.com
}

\begin{abstract}
The results of the studies of adaptive algorithm of image comparison in correlation-extreme navigation system (CENS) of aircrafts are presented in this article. The possibility of reducing the influence of impulse interference, which occurs on the viewing surface of reference area, with the help of algorithmic processing, is shown. The results of the statistical sampling of adaptive algorithm, which allows performing the selection of reference objects in conditions of influence of impulse interference, are given.
\end{abstract}

Key words: Adaptive algorithm, correlation-extreme navigation systems, viewing surface, impulse interference.

\section{INTRODUCTION}

Navigation of aircraft, which uses the correlation-extreme systems (CENS), in conditions of changes of the state of viewing surface (VS), which is caused by heavy precipitation events, for example, can disturb the process of locating the aircraft. It is based on the primary processing sensors' perception of water spills, for example, as new structural elements of VS. Taking into consideration their low brightness temperature, the water spills, for example, can be perceived by the radiometric CENS as the objects of sharp contrast to the typical surfaces, which have the higher brightness temperature values. Furthermore, the new borders between the existing objects and water spills will be visible in the current images of optoelectronic systems. The discrepancies, caused by precipitation events, will be taken by the system and will cause the inability of generation of unimodal decision function (DF) as a result of comparison between the current image (CI) and reference image (RI). In this regard the necessity of development of secondary processing algorithm occurs, the use of which will allow ensuring the needed accuracy of aircraft localization in conditions of powerful impulse interference, causing the distortion of $\mathrm{CI}[1,2,3,10]$.

\subsection{Problem analysis}

In order to ensure the high accuracy of the navigation of aircraft equipped with CENS, the works [4, 5, 6, 7] propose to create optimal RIs. The results of research on the formation of the DF under the influence of the environment on the propagation of working signals are presented in the work [8].In [9] it is proposed to use the Hough transformation to localize the contours of the objects in the images. The estimates of the influence of scale distortions on the formation of the DF are provided in [3].

However, the proposed solutions are not related to the assessment of the possible impact of powerful impulse interference on the end result of CENS operation. One of the possible ways of solving the problem of navigation of aircraft under conditions of the effect of intentional and unintentional interference on the VS is the application of adaptive algorithms of DF formation, in which by setting a quantization threshold for estimating the mean value of the background and converting the CI into a binary image, it is possible to select the brightest object used as a reference object.

The object of the article is to investigate an adaptive algorithm for binary images comparison.

\section{MAIN MATERIAL}

2.1 The description of the adaptive algorithm of image comparison

An adaptive algorithm involves the iterative processing of information about a measured parameter with a variable threshold until a single-valued solution is obtained. Commonly, the brightness is taken as a measured parameter in CENS, it varies according to the electrophysical 
characteristics of surfaces and backgrounds. Threedimensional objects located by the CENS sensors at different angles can add a significant value to the absolute brightness values.

The decision is made according to a certain rule (criterion). We can select a rule that consists of the following: a fragment of the current image $\mathbf{H}^{j} \subset \mathbf{H}$, for which when comparing $\mathrm{CI}$ and RI the condition is met:

$$
R_{j}=\inf _{i} R_{i}
$$

is declared to be completely identical to the RI. The index i, depending on the number of fragments displaced with respect to one another in a frame $\mathbf{H}$, can take $\mathbf{M}$ values. If more than one fragment coincides on criterion (1), no decision shall be taken.

When comparing the fragments of binary CI $\mathbf{H}^{i}$ with the binary RI, the decisive rule by which the fragment of CI is declared to coincide with RI is as follows:

$$
\mathrm{z}_{\mathrm{j}}=\sup _{\mathrm{i} \in \overline{0, M}} \mathrm{z}_{\mathrm{i}}
$$

Based on the general principles of the iterative process organization, with regard to CENS, we will form the description of the images of the viewing surface, in which values 1 represent objects and 0 are the backgrounds.

\subsubsection{The input data for the description of VS images}

The VS CI $\mathbf{S}_{\mathrm{CI}}$, generated by the CENS sensors shall be expressed via the original image (OI) $\mathbf{S}_{\mathrm{OI}}$, which shall be assumed to be undistorted and noise-free. These images are generally a matrix of the values of brightness of objects and VS backgrounds in resolution elements:

$$
\mathrm{S}_{\mathrm{CI}}=\mathrm{S}_{\mathrm{OI}}=\|\mathrm{S}(\mathrm{i}, \mathrm{j})\|
$$

where

$$
S(i, j)=\left\{\begin{array}{l}
S_{v}(i, j) \text {, at } S(i, j) \in S_{v} \\
S_{w}(i, j) \text { at } S(i, j) \in S_{w}
\end{array}\right.
$$

$S_{v}(i, j)$ is the brightness of $(i, j)$-th element of $v$-th object in the image;

$S_{w}(i, j)$ is the brightness of $(i, j)$-th element of $w$-th object in the image;

$\mathrm{V}$ and Ware the number of objects and backgrounds in the OI.

The impulse interference occurring on the VS shall be represented in the CI as $\mathbf{S}_{\rho}$. Hereinafter, let us assume that the signal density on the CI from impulse interference has the form of:

$$
w_{\rho}(S)= \begin{cases}\lambda e^{\lambda\left(S_{w}-S_{\rho}\right)}, & S \leq S_{\rho} \\ 0, & S>S_{\rho}\end{cases}
$$

where

$$
\lambda=\frac{\mathrm{D}_{\mathrm{e}}}{\mathrm{D}_{0}\left(\mathrm{~S}_{\mathrm{w}}-\mathrm{S}_{\rho}\right)}
$$

$\mathrm{D}_{\mathrm{e}}$-the diameter of resolution cell on the VS;

$\mathrm{D}_{0}$-the average diameter of impulse interference on the VS.

Let us make the notations:

$\mathrm{F}_{0}$-the general number of pixels of $\mathrm{CI}$;

$\mathrm{F}_{\mathrm{v}}$-the number of pixels corresponding to the object in the image;

$\mathrm{F}_{\mathrm{w}}$-the number of pixels corresponding to the background of the image;

$\mathrm{F}_{\rho}$-the number of pixels corresponding to the impulse interference in the image.

When

$$
\mathrm{F}_{\rho}+\mathrm{F}_{\mathrm{v}}+\mathrm{F}_{\mathrm{w}}=\mathrm{F}_{0}
$$

The expression (5) describes a sample $\mathrm{F}_{0}$ comprising three groups $\omega_{\mathrm{i}}$ corresponding to the background $\omega_{\mathrm{w}}$, interference $\omega_{\rho}$ and object $\omega_{v}$.

The sampling density can then be presented as follows:

$$
\mathrm{w}(\mathrm{S})=\sum_{\mathrm{i}=1}^{3} \mathrm{p}_{\mathrm{i}} \mathrm{w}_{\mathrm{i}}(\mathrm{S}),
$$

where $\mathrm{p}_{\mathrm{i}}=\frac{\mathrm{F}_{\mathrm{i}}}{\mathrm{F}_{0}}, \mathrm{i}=1,2,3-\mathrm{a}$ priori probabilities of groups; $\mathrm{w}_{\mathrm{i}}(\mathrm{S})=\mathrm{w}\left(\mathrm{S} \mid \omega_{\mathrm{i}}\right)$-conditional densities of probability of random value $S$ provided that $S$ belongs to the group $\omega_{i}$.

As a result, for the selection of signals on the CI from the object on the VS, let us present a sample (3) in two groups: 1 group - signals from the object;

2 group - other signals.

\subsubsection{The organization of iterative process}

To determine the initial value of the threshold $l^{0}$ there is an estimate of the mean value $\widehat{S}_{w}$ for which the CI is converted to a binary image $\mathbf{H}^{0}$. Comparing the CI to the RI according to the rule (2), a matrix $\left\|z_{\mathrm{ij}}^{0}\right\|$ is formed and a set of values is determined:

$$
\mathbf{M}^{0}=\left\{(\mathrm{k}, \mathrm{l}) \in \overline{1, \mathrm{~N}_{1}} \times \overline{1, \mathrm{~N}_{2}} \mid \mathrm{z}_{\mathrm{kl}}=\max _{\mathrm{i}, \mathrm{j}} \mathrm{z}_{\mathrm{ij}}\right\} .
$$

The maximum value of this population may differ from $F_{v}$ (it is probable that $\mathrm{z}_{\max }^{0}<\mathrm{F}_{\mathrm{v}}$ ).

In case when $\mathbf{M}^{0}=\{1(m, l)\}$, it is decided that the coordinates of the pivot element of the object are equal to the CI values $m, l$. 
If no decision is taken, a three-stage iterative process is organized.

In the first stage, the initial value of the threshold $l^{0}$ is replaced by $l^{1}$.

In the second stage, a new $\mathrm{CI} \mathbf{H}^{1}$ is formed relative to the threshold $l^{1}$.

In the third step, the value matrix $\left\|z_{i j}^{1}\right\|$ for $\mathrm{CI} \mathbf{H}^{1}$ is calculated and the sum of $\mathbf{M}^{1}$ values is determined.

The organization of the iterative process necessitates the determination of the size of the iterative step and its sign. The step selection of each iteration must be made with the respect to the following: CI $\mathbf{H}^{i}$ should not drastically differ from $\mathbf{H}^{i-1}$ at the next $i$-th iteration.

The search is carried out up to the moment when the highest value of the result of the comparison of the images corresponding to the perfect match of the CI and RI are found according to the criterion:

$$
\mathrm{G}_{\mathrm{j}}=\sup _{\mathrm{i} \in \overline{0, \mathrm{U}}} \mathrm{G}_{\mathrm{i}} \text {. }
$$

The set $\mathbf{G}_{i}$, in which the largest number of units is written is taken as the result of the selection of the target object.The cell $\mathrm{G}_{\mathrm{j}}(\mathrm{i}, \mathrm{j})$ with the largest number of units allows to determine the number of the step-by-step comparison by rows and columns of the set in which the target object is selected.

Thus, a decision is made to select the target object in the frame of the CI.

\subsection{The results of statistical sampling of adaptive algorithm}

Test objectives: To evaluate the performance of an adaptive algorithm under different interference conditions using statistical modelling.

The test program consisted of:

1) Simulation of a noise-free CI based on the "object against background" image model;

2) Simulation of the position of impulse interference in the frame of the CI and its amplitude;

3) Simulation of the CI by applying to the noise-free CI the noise,simulating the interior noise of radiometric channels;

4) Simulation of the algorithm of selection of an object using binary RI and its modification with adaptation of the threshold;

5) Statistical tests of algorithms of both types to assess their performance for different parameter values;

6) a theoretical evaluation of the effectiveness of an nonadaptive algorithm;

7) Comparison of theoretical results with those, obtained with the help of statistical modelling.
The test procedurewas outlined as follows:

CI and RI were initially modelled based on the "object against background" model.

Let us denote by

$\mathrm{T}^{\mathrm{m}}=\left[\mathrm{T}_{\mathrm{ij}}^{\mathrm{m}}\right], \mathrm{i} \in \overline{1, \mathrm{~N}_{1}}, \mathrm{j} \in \overline{1, \mathrm{~N}_{2}}$ the presentation of CI,

and by $\mathrm{T}^{\mathrm{v}}=\left(\mathrm{T}_{1}^{\mathrm{v}}, \ldots, \mathrm{T}_{\mathrm{N}_{\mathrm{im}}}^{\mathrm{v}}\right)\left(\mathrm{N}_{\mathrm{im}}=\mathrm{N}_{1} \mathrm{~N}_{2}\right)$ its vector representation obtained by the involute of the CI set by rows.

Transitions between the element indices are performed according to the following rules:

$$
\begin{aligned}
& (\mathrm{i}, \mathrm{j}) \mathrm{ak}=\mathrm{N}_{2}(\mathrm{i}-1)+\mathrm{j} ; \\
& \mathrm{k} \mapsto\left(\mathrm{i}=\operatorname{div}_{\mathrm{N}_{2}} \mathrm{k}+1, \mathrm{j}=\bmod _{\mathrm{N}_{2}} \mathrm{k}\right)
\end{aligned}
$$

The CI matrix is assigned values $\mathrm{T}_{\phi}$, i.e.

$$
\mathrm{T}_{\mathrm{ij}}^{\mathrm{m}}=\mathrm{T} \phi, \mathrm{i} \in \overline{1, \mathrm{~N}_{1}}, \mathrm{j} \in \overline{1, \mathrm{~N}_{2}} .
$$

The binary RI of the elements $\mathrm{J}_{3}$ whose configuration is assumed to be known is defined as follows. If you put the $\mathrm{RI}$ on the CI set, then the reference or characteristic element of the target is the element with the smallest number in the CI vector representation. In other words, if $\mathrm{c}=\left(\mathrm{c}_{1}, \ldots, \mathrm{c}_{\mathrm{J}_{3}}\right)$ is the vector of the target numbers, then $c_{\mathrm{op}}=\mathrm{c}_{1}$ is the reference element number.

Let's construct the RI vector as follows:

$$
\mathrm{e}=\left(\mathrm{e}_{1}, \ldots, \mathrm{e}_{\mathrm{J}_{3}-1}\right)=\left(\mathrm{c}_{2}-\mathrm{c}_{1}, \ldots, \mathrm{c}_{\mathrm{J}_{3}}-\mathrm{c}_{\mathrm{J}_{3}-1}\right) \text {. }
$$

Then the object vector is recovered by the reference element $\mathrm{c}_{\mathrm{op}}$ number and the RI vector $\mathbf{e}$ by the relation:

$$
\mathrm{c}=\left(\mathrm{c}_{1}=\mathrm{c}_{\mathrm{op}}, \mathrm{c}_{2}=\mathrm{c}_{1}+\mathrm{e}_{1}, \ldots, \mathrm{c}_{\mathrm{J}_{3}}=\mathrm{c}_{\mathrm{J}_{3}-1}+\mathrm{e}_{\mathrm{J}_{3}-1}\right)
$$

The aim localization problem is to determine the number of the reference element, according to which with the help of the relation (8) we can find the coordinates of the reference element in the matrix representation:

$$
\mathrm{i}_{\mathrm{op}}=\operatorname{div}_{\mathrm{N}_{2}} \mathrm{c}_{\mathrm{op}}+1, \mathrm{j}_{\mathrm{op}}=\bmod _{\mathrm{N}_{2}} \mathrm{c}_{\mathrm{op}} \text {. }
$$

Let us assign elements $\mathbf{T}^{\mathbf{v}}$ with numbers $\mathrm{c}_{1}, \ldots, \mathrm{c}_{\mathrm{J}_{3}}$ the values $\mathrm{T}_{\phi-\mathrm{B}}$. Then, according to the interference model, the coordinates of each element of the CI affected by the interference are randomly given for each coordinate in accordance with the uniform law of distribution, and the amplitude of the interference is chosen based on the exponential law of probability distribution. It is provided that, in case of a disturbance on an element occupied by an object, a new drawing of the position of interference shall 
be made until it hits an element of the image corresponding to the background. This completes the formation of a noisefree $\mathrm{CI}$.

The final construction of the CI is carried out by adding to each element of the CI a random normally distributed value with zero mean and mean standard deviation $\sigma$, thus completing the simulation part of the algorithm.

The algorithm for selecting an object is the following. The limits of the RI scanning by CI are defined, i.e. the set of valid reference element coordinates such that the RI constructed from this element did not exceed the CI. For this purpose, some initial value of the support element number is given $\mathrm{k}_{\mathrm{H}}, 1 \leq \mathrm{k}_{\mathrm{H}}<\mathrm{N}_{2}$. Based on (8) it follows that $\mathrm{i}_{\mathrm{H}}=1, \mathrm{j}_{\mathrm{H}}=\mathrm{k}_{\mathrm{H}}$.

According to formula (10), a vector $\mathbf{c}$ is constructed and calculated

$$
\begin{gathered}
\mathrm{j}_{\min }=\min \left(\mathrm{j}_{\mathrm{c}_{1}}, \ldots, \mathrm{j}_{\mathrm{c}_{\mathrm{J}_{3}}}\right), \\
\mathrm{j}_{\max }=\max \left(\mathrm{j}_{\mathrm{c}_{1}}, \ldots, \mathrm{j}_{\mathrm{c}_{\mathrm{J}_{3}}}\right), \mathrm{i}_{\mathrm{c}_{\mathrm{J}_{3}}}, \mathrm{j}_{\mathrm{c}_{\mathrm{J}_{3}}} .
\end{gathered}
$$

Then by the formula:

$$
\begin{aligned}
& \mathrm{J}_{\text {min }}=\mathrm{i}_{\mathrm{H}}+1-\mathrm{j}_{\min } ; \mathrm{J}_{\max }=\mathrm{N}_{2}+\mathrm{i}_{\mathrm{H}}-\mathrm{j}_{\max } ; \\
& \mathrm{I}_{\min }=1 ; \mathrm{I}_{\max }=\mathrm{N}_{1}+\mathrm{i}_{\mathrm{H}}-\mathrm{i}_{\mathrm{c}_{\mathrm{J}_{3}}}
\end{aligned}
$$

the horizontal and vertical limits of the RI scanning are defined.

Next is an estimate of the mean value of the background $\hat{\mathrm{T}}_{\phi}$, the CI is converted to binary relative to some threshold 1 and by scanning the RI the set of the decision function is calculated.

If the maximum is the single one, a decision is made on the selection of the object and the position of the coordinates $\left(\hat{\mathrm{i}}_{\mathrm{op}}, \hat{\mathrm{j}}_{\mathrm{op}}\right)$ is determined according to the position of the maximum of decision function. If the maximum is reached on several elements at once, in case of the non-adaptive algorithm the decision is made not to take a decision, and in case of an adaptive algorithm, the threshold for converting the CI into a binary image will be changed in such a manner that to reduce the number of extreme elements to one element.

Statistical tests of the algorithm are then carried out, i.e. a new implementation of the CI is formed and a selection algorithm of the object is started.

Multiple runs of the algorithm compute the probability of the correct localization of an object as the relation of the number of correctly selected cases to the total number of algorithm starts in the test series.
For an non-adaptive algorithm, each series calculates theoretical efficiency estimates based on the formula:

$$
\mathrm{P}_{\mathrm{co}}=\sum_{\mathrm{j}=1}^{\mathrm{J}_{3}} \mathrm{C}_{\mathrm{J}_{3}}^{\mathrm{j}}(1-\alpha)^{\mathrm{j}} \alpha^{\mathrm{J}_{3}-\mathrm{j}}\left[\sum_{\mathrm{k}=0}^{\mathrm{j}-1} \mathrm{C}_{\mathrm{J}_{3}}^{\mathrm{k}} \beta^{\mathrm{k}}(1-\beta)^{\mathrm{J}_{3}-\mathrm{k}}\right]^{\mathrm{K}} \text {. }
$$

Results of statistical tests.

The algorithms were tested with and without adaptation of the threshold of conversion of RI to binary image. The parameters of the simulated CI were taken equal to: $\mathrm{N}_{1}=\mathrm{N}_{2}=8 ; \mathrm{N}_{\text {im }}=64$;

coordinates of the reference element in CI $\mathrm{k}_{\mathrm{op}}=27\left(\mathrm{i}_{\mathrm{op}}=4 ; \mathrm{j}_{\mathrm{op}}=3\right) ; \quad \sigma=1 \mathrm{~K} ;$ background temperature $\mathrm{T}_{\phi}=270 \mathrm{~K}$;

RI parameters: number of elements $\mathrm{J}_{3}=3$; RI vector $\mathbf{e}=(1,7)$.
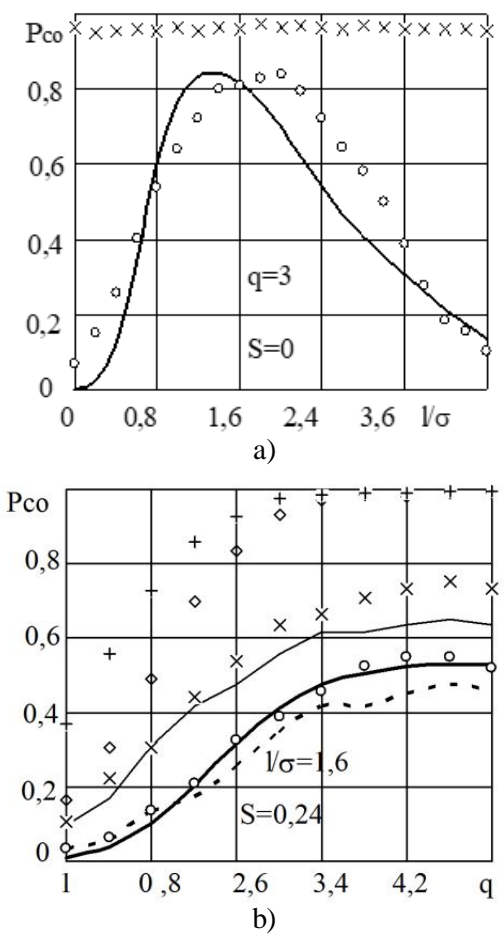

Figure 1: The correspondence $\mathrm{P}_{c o}(1 / \sigma)$

In Figure $1 \mathrm{a}, \mathrm{b}$ the correspondence of the probability of the correct localization of the target with the value of the relative threshold $l^{\prime}=1 / \sigma$ at the value of the interference parameter $\mathrm{S}=\mathrm{J}_{2} / \mathrm{J}_{1}=0$ (a) and $\mathrm{S}=0,24$ (b) for the fixed value of the signal-noise ratio $q=B / \sigma=3$. Theoretical curves for the algorithm without adaptation of the threshold are indicated by continuous lines, corresponding results of statistical tests are shown by circles and in the case of adaptive algorithm by crosses.

The analysis of the graphs in Figure 1 shows that:

1) In general, the theoretical curve reflects correctly the course of the experimental relation; 
2) The maximum experimental curve relative to the theoretical one is slightly shifted towards the higher relative threshold values, with the displacement decreasing with the increase of the interference;

3) The efficiency of the adaptive algorithm is virtually independent of the threshold value and significantly exceeds the efficiency of the algorithm without adapting the threshold.

In constructing the graphs presented in Figure. 1, implementations $\mathrm{N}_{\mathrm{z}}=1000$ were processed to form one probability count.

In Figure $2 \mathrm{a}, \mathrm{b}$ graphs of the correspondence of the probability of the correct selection of an object with the signal-noise ratio in the image at a fixed value of the relative threshold $l^{\prime}=1,6$ and the values of the parameter $\mathrm{S}=0$ (a) and $\mathrm{S}=0,24$ (b) are presented.

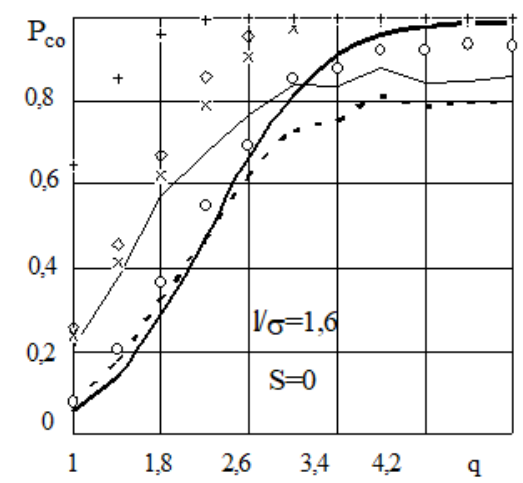

a)

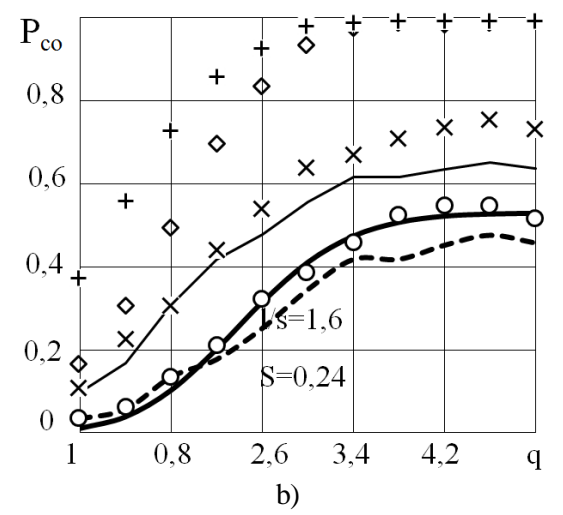

Figure 2: The correspondence $\mathrm{P}_{\text {co }}(1 / \sigma)$

The results of the statistical tests of the non-adaptive and adaptive algorithms for the purpose of the three elements are still represented by circles and crosses " $\times$ ". Diamonds and pluses show the results of the tests of the same algorithms in the same diagrams, but with respect to an object, occupying $\mathrm{J}_{3}=21$ element with the RI vector

$$
\mathbf{e}=(1,1,1,1,4,1,1,1,1,4,1,1,1,1,4,1,1,6,1,1) .
$$

The analysis of graphs shows a satisfactory agreement between theoretical studies and statistical tests of the algorithm without the adaptation of the threshold, as well as substantial superiority in the efficiency of the adaptive algorithm. As the number of elements of an object increases, the characteristics of both algorithms improve, but this conclusion is only valid if the object does not go beyond the frame of the CI, otherwise the efficiency drops rapidly.

A dotted line and a thin continuous line are shown on the graphs as the results of the tests of non-adaptive and adaptive algorithms, respectively, when the position of the pivot element of an object consisting of three elements, was not fixed, but was randomly set in accordance with the uniform law of probability distribution within the taken limits $\mathrm{I}_{\min }, \mathrm{I}_{\max }, \mathrm{J}_{\min }, \mathrm{J}_{\max }$.

It should be noted that there has been a marked reduction in the efficiency of algorithms in the field of large values of signal-to-noise ratios.

During the statistical tests of the algorithm with the adaptation of the threshold, it was noted that the iterative procedure of ascertaining the mean value of the background greatly reduces the speed of the algorithm, with little impact on its efficiency. Since the use of the principle of adaptation reduces the requirements for accuracy in estimating the mean of the background, it is sufficient to use the method of rough estimation of the mean of the background, which allows to increase the speed of the algorithm several times without losing its efficiency.

During the statistical tests of algorithms, the probability of the correct localization of the target was assessed based on the results $\mathrm{N}_{\mathrm{z}}=1000$ tests. But in the most unfavorable case, corresponding to $\mathrm{P}_{\mathrm{co}}=1 / 2$, for probability belief $\beta=0,9$ the confidence interval is $[0,474 ; 0,526]$.

\section{CONCLUSION}

The research has established the good agreement of the results of theoretical studies and the statistical sampling of the algorithm without the threshold adaptation as well as the substantial advantage in the effectiveness of the adaptive algorithm. It is found that with an increase in the number of elements of an object, the characteristics of both algorithms improve, but this is only true if the object does not go beyond the frame of the CI.

The iterative procedure of updating the mean of the background deteriorates substantially the response speed of the algorithm with little impact on its effectiveness.Concurrently, the use of adaptation principle allows to lower the requirements to the estimating the mean of the background, for this procedure it suffice to use the method of raw estimation of the mean of the background, which allows to increase the response speed of the algorithm several times without losing its efficiency.

\section{REFERENCES}

1. V. Antyufeev. Matrix radiometric correlation-
extreme navigation systems for aircraft: 
monographUkraine, Kharkov: KhNU V.N. Karazin, $372 \mathrm{p}$.

2. A. Sotnikov, V. Tarshyn, N. Yeromina, S. Petrov, N. Antonenko. A method for localizing a referenceobject in a current image with several bright objects, Eastern-European Journal of Enterprise Technologies. 2017. Vol. № 9 (87). pp. 68-74. DOI: 10.15587/1729-4061.2017.101920.

3. N.Yeromina, S. Petrov, A. Tantsiura, M. Iasechko, V. Larin. Formation of reference images and decisionfunction in radiometric correlationextremal navigation systems. Eastern-European Journal ofEnterprise Technologies. - 2018. Vol.4, No.9 (94). - pp. 27-35. DOI: 10.15587/17294061.2018.139723.

4. O.Tymochko, A.Ttystan , V.Ushan , N.Yeromina , O.Dmitriiev, V.Mazharov, I.Padalka , I.Hannoshyna, I. Masik , A. ZazirnyiThe Synthesis of the Reference Image and Algorithms for Vehicle Navigation Systems, JETER,8(3), 2020, pp. 853-858. doi: 10.30534/ijeter/2020/40832020.

5. S. Trehubenko, L. Berkman, N. Yeromina, S. Petrov, Y. Bryzhatyi, H. Kovalov, V.Dachkovskyi, L.MikhailovaThe Operation of Detection Systems in Conditions of Contrast Decrease of Ground Objects, JETER, 8 (1), 2020, pp. 208-212, doi: 10.30534/ijeter/2020/28812020

6. N.Yeromina, S. Petrov, A. Tarasenko, N. Antonenko, O. Semenenko, O. Pavlovskyi, M. Sliusarenko, V. TelehinThe Use of Strong and Ordinal Scales during the Synthesis of Reference Images for Vehicle Correlation-Extreme, IJATCSE, 9 (2), 2020, pp. 2343-2349, doi: 10.30534/ijatcse/2020/218922020

7. N. Yeromina, S. Petrov, N. Antonenko, I. Vlasov, V. Kostrytsia, V. KorshenkoThe Synthesis of the Optimal Reference Image Using Nominal and Hyperordinal Scales, JETER, 8 (5), 2020, pp. 20802084, doi: 10.30534/ijeter/2020/98852020

8. O. Liashko, V. Klindukhova, N.Yeromina, T.Karadobrii, O.Bairamova, A. DoroshevaThe Criterion and Evaluation of Effectiveness of Image Comparison in Correlation-Extreme Navigation Systems of Mobile Robots, JETER, 8 (6), 2020, pp. 2841-2847, doi: 10.30534/ijeter/2020/ 97862020

9. N.Yeromina, V. Samoilenko, D. Chukanivskyi, O. Zadkova, O. Brodova, O. Levchenko The Method of Iterative Formation of Selective Reference Images, JETER, 8 (7), 2020, pp. 3753-3759, doi: 10.30534/ijeter/2020/138872020

10. Smelyakov, K., Hvozdiev, M., Chupryna,A., Sandrkin, D., Martovytskyi, V.Comparative Efficiency Analysis of Gradational Correction Models of Highly Lighted Image //2019 IEEE International Scientific Practical Conference Problems of Infocommunications, Science and Technology (PIC S \&amp; T). - IEEE, 2019.- C. 703-708. 